\title{
Neutrophils Compromise Retinal Pigment Epithelial Barrier Integrity
}

\author{
Jiehao Zhou, ${ }^{1}$ Shikun He, ${ }^{1,2,3}$ Ning Zhang, ${ }^{4}$ Christine Spee, ${ }^{1,3}$ Peng Zhou, ${ }^{1,3}$ \\ Stephen J. Ryan, ${ }^{1,3}$ Ram Kannan, ${ }^{1,3}$ and David R. Hinton ${ }^{1,2,3}$ \\ ${ }^{1}$ The Arnold and Mabel Beckman Macular Research Center at the Doheny Eye Institute, University of Southern California, \\ Los Angeles, CA 90033, USA \\ ${ }^{2}$ Department of Pathology, Keck School of Medicine, University of Southern California, Los Angeles, CA 90033, USA \\ ${ }^{3}$ Department of Ophthalmology, Keck School of Medicine, University of Southern California, Los Angeles, CA 90033, USA \\ ${ }^{4}$ Department of Pharmaceutical Sciences, School of Pharmacy, University of Southern California, Los Angeles, CA 90033, USA
}

Correspondence should be addressed to David R. Hinton, dhinton@usc.edu

Received 8 July 2009; Revised 15 October 2009; Accepted 19 November 2009

Academic Editor: Karl Chai

Copyright () 2010 Jiehao Zhou et al. This is an open access article distributed under the Creative Commons Attribution License, which permits unrestricted use, distribution, and reproduction in any medium, provided the original work is properly cited.

\begin{abstract}
We hypothesized that neutrophils and their secreted factors mediate breakdown of the integrity of the outer blood-retina-barrier by degrading the apical tight junctions of the retinal pigment epithelium (RPE). The effect of activated neutrophils or neutrophil cell lysate on apparent permeability of bovine RPE-Choroid explants was evaluated by measuring $\left[{ }^{3} \mathrm{H}\right]$ mannitol flux in a modified Ussing chamber. The expression of matrix metalloproteinase- (MMP-) 9 in murine peritoneal neutrophils, and the effects of neutrophils on RPE tight-junction protein expression were assessed by confocal microscopy and western blot. Our results revealed that basolateral incubation of explants with neutrophils decreased occludin and ZO- 1 expression at 1 and 3 hours and increased the permeability of bovine RPE-Choroid explants by $>3$-fold $(P<.05)$. Similarly, basolateral incubation of explants with neutrophil lysate decreased ZO-1 expression at 1 and 3 hours $(P<.05)$ and increased permeability of explants by 75\%. Further, we found that neutrophils prominently express MMP-9 and that incubation of explants with neutrophils in the presence of anti-MMP-9 antibody inhibited the increase in permeability. These data suggest that neutrophil-derived MMP-9 may play an important role in disrupting the integrity of the outer blood-retina barrier.
\end{abstract}

\section{Introduction}

The outer blood-retinal barrier (BRB) is a specialized transport barrier between the vascular choriocapillaris and the neural retina that regulates the exchange of fluid, nutrients, and waste products. Breakdown of the outer BRB is a feature of many blinding retinal disorders such as proliferative vitreoretinopathy (PVR), uveal-retinal inflammation, diabetic retinopathy, and age-related macular degeneration (AMD) [1-4]. The side effects of some therapeutic interventions (e.g., cryotherapy and laser photocoagulation) include a breakdown of the outer BRB [1-4].

Because the choriocapillaris is fenestrated, the actual barrier function of outer $\mathrm{BRB}$ is mediated by the monolayer of retinal pigment epithelial (RPE) cells [5]. Apical tight junctions joining adjacent RPE maintain the continuity of the barrier between cells and are critical for maintaining the normal polarized functions of the RPE monolayer [6]. RPE tight junctions consist of a complex of proteins including claudins, occludin, and zonula occludens- (ZO-) $1[7,8]$. While occludin is a transmembrane protein, and major structural component of the tight junction, $\mathrm{ZO}-1$ is a peripheral adaptor protein, linking occludin with the actin cytoskeleton. Therefore, expressions of ZO-1 and occludin are considered as useful markers of tight junction structure between RPEs [9]. The mechanisms that need to be considered underlying the breakdown of the outer BRB include attenuation and disruption of intercellular tight junctions or death of RPE.

Neutrophils, which are the most abundant leukocytes in the circulation, respond rapidly to inflammatory or infectious stimuli. During acute inflammation, neutrophils 
interact with endothelial cells through adhesion molecules, leading to disassembly of endothelial tight junctions and permitting neutrophil extravasation $[10,11]$. Neutrophils also secrete numerous preformed bioactive proteins, such as matrix metalloproteinases (MMPs) [12] which degrade junctional proteins including tight junction components, thus facilitating the breakdown of the vascular barrier. The possibility that neutrophils could play a role in modulating the outer BRB in retinal disease is supported by the finding of increased number of neutrophils in the choriocapillaris of patients with diabetes and in the choriocapillaris of streptozotocin-induced experimental diabetes in mice [1315]. Accumulation of neutrophils is also associated with proliferative vitreoretinopathy [3] and uveitis, conditions in which the outer BRB is compromised [4]. As well, we have previously shown that neutrophils promote laser-induced choroidal neovascularization (CNV) in mice, which is a wellestablished model for study of the pathogenesis of the wet form of AMD [16]. Both in vitro and in vivo studies have demonstrated that under pathologic conditions, RPEs secrete a number of chemokines, including IL-8 [17], which is responsible for the recruitment/accumulation of neutrophils. In the presence of inflammatory mediators, such as tumor necrosis factor- (TNF-) $\alpha$, RPE cells also have been shown to secrete MMP-9, which could exacerbate effects of neutrophilderived MMP-9 [18]. In the vasculature, neutrophils interact with endothelial cells through adhesion molecules, such as CD18/ICAM-1. Increased neutrophil adhesion induces enhanced endothelial injury through FAS/FASL interaction, which is associated with increased endothelial apoptosis and subsequent BRB permeability [13]. Therefore, the hypothesis of the current study was that neutrophils and their secreted factors mediate breakdown of the integrity of the outer BRB by degrading the apical tight junctions of the RPE.

In the present study, we examined the effects of neutrophils on the permeability of the outer BRB in RPEChoroid explants in vitro and the expression of tight junction proteins. Furthermore, we evaluated the role of MMP-9 in this process.

\section{Methods}

2.1. Animals. C57BL/6 male mice were purchased from the National Cancer Institute (Frederick, MD). Mice between 6 to 8 weeks old were fed standard laboratory chow and kept in an air-conditioned room on a 12-hour light /12-hour dark cycle. All procedures were performed in compliance with the Keck School of Medicine Institutional Animal Care and Use Committee approved protocols and the Association for Research in Vision and Ophthalmology (ARVO) Statement for the Use of Animals in Ophthalmic and Vision Research.

2.2. Preparation of Murine Neutrophils. A neutrophilenriched population was isolated from murine peritoneal exudates after glycogen challenge $[19,20]$. Briefly, oyster glycogen (Sigma, St. Louis, MO) was dissolved in sterile
$0.9 \% \mathrm{NaCl}$ to a final concentration of $1 \mathrm{mg} / \mathrm{mL}$. Mice received i.p. injection of $1 \mathrm{~mL}$ of the prepared solution and the peritoneal exudates were harvested 4 hours later. The exudate cells comprise approximately 90\%-95\% neutrophils as determined by their characteristic morphology under the microscope and by flow cytometry using anti-Ly6G (Becton Dickinson, Pharmingen, San Diego, CA), a specific antineutrophil antibody (data not shown). The neutrophilenriched population was washed 3 times by centrifugation at $300 \mathrm{~g}$ for 5 minutes and resuspended in PBS to remove residual glycogen. To obtain soluble neutrophil extract, the peritoneal exudate containing 90\%-95\% neutrophils was sonicated three times for 10 seconds on ice with a Branson sonifier (Model 185) and was centrifuged at 12,000 rpm for 10 minutes at $4^{\circ} \mathrm{C}$.

2.3. RPE-Choroid Explant Culture and Treatment. Fresh bovine eyes were obtained from Manning's Beef L.L.C. (Pico Rivera, CA). The eyes were immersed in Hanks' balanced salt solution (Irvine Scientific, Santa Ana, CA) containing $1 \%$ penicillin and $1 \%$ streptomycin for 10 minutes. Under a dissecting microscope in sterile conditions, the vitreous humor and neural retina were carefully removed without disturbing the RPE layer. The eyes were then cut into 2 to $3 \mathrm{~mm}^{2}$ pieces, each with an intact RPE layer, choroid, and sclera. The sclera was carefully removed and the RPEChoroid explants were immediately placed in high-glucose Dulbecco's modified Eagle's medium (Irvine Scientific) supplemented with penicillin $\mathrm{G}(100 \mathrm{U} / \mathrm{mL})$, L-glutamine $(0.292 \mathrm{mg} / \mathrm{mL})$, and $1 \%$ fetal bovine serum (FBS; Irvine Scientific). Explants were cultured with or without murine neutrophils or neutrophil lysate at 8 time points up to 3 hours for mannitol transport experiments, and at 1 and 3 hours for tight junction protein expression by confocal microscopy, and 3 hours for tight junction protein expression by western blot. The viability of explants has been demonstrated to be up to 72 hours [9]. In experiments evaluating effects of anti-MMP-9 antisera, goat antimouse MMP-9 inhibitory IgG (sc-6841; Santa Cruz Biotechnology, Inc., Santa Cruz CA), was mixed with the neutrophil suspension prior to adding to the explant, at a dose suggested by the manufacturer $(2 \mu \mathrm{g} / \mathrm{mL})$. Normal goat IgG (sc-2028; Santa Cruz Biotechnology, Inc.) at the same concentration was used as a negative control.

\subsection{Mannitol Transport in Bovine RPE-Choroid Explants Mounted in Ussing Chamber. The bovine RPE-Choroid explants were mounted in a custom designed Ussing cham- ber as previously described [21]. Briefly, the retina-RPE- choroid explants were placed on a nylon mesh (Becton Dick- inson Discovery Labware, Bedford, MA), with the retinal side up. The RPE-choroid was mounted in a custom designed Ussing chamber with an exposed area $(A)$ of $1.0 \mathrm{~cm}^{2}$. The RPE-choroid preparations mounted in a clamping chip were placed between two chamber halves. The chamber held $6 \mathrm{ml}$ bicarbonated Ringer's solution (BRS) on each side of the tissue and was water-jacketed to keep the temperature within the chamber at $37^{\circ} \mathrm{C}[22]$. All experiments were}


performed under short-circuit condition using an automatic voltage-clamp device (558C-5; Bioengineering Department, University of Iowa, Iowa City, IA), as previously described [23]. Basolateral to apical mannitol flux measurement was initiated by adding $\left[{ }^{3} \mathrm{H}\right]$ mannitol $(2.0 \mu \mathrm{Ci} / \mathrm{mL}$, Moravek Biochemicals, Brea, CA) to the basolateral donor fluid. The neutrophils or neutrophil lysate were added at the basolateral side of the RPE-Choroid explant. A $0.6 \mathrm{ml}$ aliquot was collected from the receiver fluid every 15-30 minutes for a total of 3 hours for assay of radioactivity in a liquid scintillation counter (Beckman Instruments, Fullerton, CA). The removed aliquot was immediately replaced with an equal volume of fresh BRS buffer. The area normalized permeation amount $\left(Q, \mathrm{~mol} / \mathrm{cm}^{2}\right)$ for mannitol was calculated using the following equation. $Q=$ total counts per minute $(\mathrm{cpm})$ in receiver fluid $\times$ specific activity $\left.(\mathrm{mol} / \mathrm{cpm})] / A\left(\mathrm{~cm}^{2}\right)\right]$.

Area normalized unidirectional fluxes $\left(\mathrm{J}\right.$, moles $/ \mathrm{cm}^{2} \cdot \mathrm{h}$ ) were then estimated from the slope of a plot of cumulative amount of penetrate appearing in the receiver fluid versus time. The apparent permeability coefficient $\left(P_{\text {app }}\right)$ was calculated by further normalizing the flux against the initial substrate concentration in the donor fluid.

2.5. Confocal Immunofluorescence Microscopy. The expression and localization of the tight junction proteins occludin and ZO-1 in explants were examined by immunofluorescent confocal microscopy. Explants were fixed in 2\% paraformaldehyde in PBS for 15 minutes at room temperature, permeabilized in $0.2 \%$ Triton X-100 in PBS for 15 minutes, and incubated with the primary antibody antiZO-1 (1 : 100; Chemicon, Temecula, CA) or antioccludin (1 : 100; Zymed Laboratories, South San Francisco, CA) for 60 minutes at $4^{\circ} \mathrm{C}$ and then with the secondary antibody, rhodamine-conjugated goat antirabbit $(1: 400$; Chemicon) for 30 minutes at $4{ }^{\circ} \mathrm{C}$. The explants were washed in PBS, mounted, and examined with a Zeiss LSM 510 confocal microscope (Carl Zeiss, Thornwood, NY) with a 40x oilimmersion objective using identical settings for laser power, pinhole size, and detector sensitivity. Quantitation of ZO-1 and occludin immunofluorecence area was done using the LSM 510 image software version 3.2. to calculate percent fluorescently stained area normalized to untreated control samples. All threshold values were identical in order to collect comparable results. Data were represented as positive area/40x objective field.

To determine the expression of MMP-9 in neutrophils by confocal microscopy, the neutrophil-enriched population was centrifuged onto glass slides. The cytospin slides were permeabilized with $0.1 \%$ Triton X-100 and fixed in acetone for 10 minutes at room temperature. The expression of MMP-9 was identified using goat antimouse MMP-9 antibody (Santa Cruz Biotechnology, Santa Cruz, CA) and Rhodamine antimouse secondary antibody (Chemicon). The slides were mounted in VECTASHIELD mounting medium with 4'6-Diamidino-2-phenylindole (DAPI) nuclear counterstain (Vector Laboratories, Burlingame, CA) and examined under a Zeiss LSM510 confocal microscope.
2.6. Western Blot Analysis. The explant was homogenized in $100 \mu \mathrm{L}$ modified RIPA buffer $(50 \mathrm{mM}$ Tris- $\mathrm{HCl}(\mathrm{pH}$ 7.4 ), $1 \%$ Triton X-100, $0.2 \%$ sodium dodecyl sulfate (SDS), $1 \mathrm{mM}$ dithiothreitol, $2 \mathrm{mM}$ ethylene glycol tetraacetic acid (EGTA), $4 \mathrm{mM}$ ethylene diamine tetraacetic acid (EDTA), $2 \mathrm{mM}$ sodium orthovanadate, and $100 \mathrm{mM} \mathrm{NaCl}$ ) with fresh protease inhibitors, including $0.2 \mathrm{mM}$ phenylmethylsulfonyl fluoride (PMSF) and $10 \mu \mathrm{g} / \mathrm{mL}$ each of aprotinin, pepstatin $\mathrm{A}$, and soybean trypsin inhibitor (Sigma, St. Louis, MO). Samples were incubated at $4^{\circ} \mathrm{C}$ for 30 minutes to solubilize proteins, and insoluble materials were pelleted by centrifugation at $14,000 \mathrm{rpm}$ for 10 minutes. Protein concentration was determined with a protein assay (BioRad, Richmond, CA), and equal total protein content was loaded into 7.5\% SDS-polyacrylamide gels (SDS-PAGE; BioRad). Proteins were transferred to polyvinylidene difluoride membranes (Millipore, Bedford, MA), blocked by $1 \%$ bovine serum albumin for 60 minutes, and incubated with the primary antibodies of anti-ZO-1 (1 : 1000; Chemicon), anti-occludin (1 : 1000; Zymed Laboratories), followed by horseradish peroxidase-conjugated secondary antibody. The membranes were then washed and developed with the addition of a chemiluminescence detection kit (Amersham Pharmacia Biotech, Piscataway, NJ). For the protein loading control, the same blot was reprobed with anti-GAPDH antibody (Chemicon). Quantitation of occludin or ZO-1 pixel density was achieved by densitometry with normalization to GAPDH.

2.7. Cell Viability Assay. Cell viability was assessed by propidium iodide (PI) staining after RPE cells were exposed to neutrophils or medium; RPE cells treated with $20 \mu \mathrm{M}$ C2-ceramide (Sigma) underwent cell death and served as a positive control after PI staining [24]. Pure cultures of human RPE cells (passage 3) were derived as previously described, trypsinized and suspended in PBS [25]. Nonspecific binding was minimized by blocking with $1 \%$ bovine serum albumin (Sigma) for 20 minutes at room temperature before staining. Cells were stained with PI for 30 minutes on ice, washed three times with PBS containing 0.5\% bovine serum albumin, and 5000 cells/sample were analyzed using a FACStar flow cytometer and Cellquest Pro software (Becton Dickinson, Mountain View, CA). Viable cells were PI negative.

2.8. Statistics. All experiments were carried out three times (except for apparent permeability experiments using neutrophil lysates which were performed twice); statistical analyses were performed using the Student's $t$-test. Values reported in figures represent mean \pm standard deviation. Accepted level of significance for all tests was $P<.05$.

\section{Results}

3.1. Neutrophils Compromise the RPE Barrier Integrity. The effect of neutrophils on RPE barrier integrity was assessed by measuring RPE-Choroid explant permeability using a 


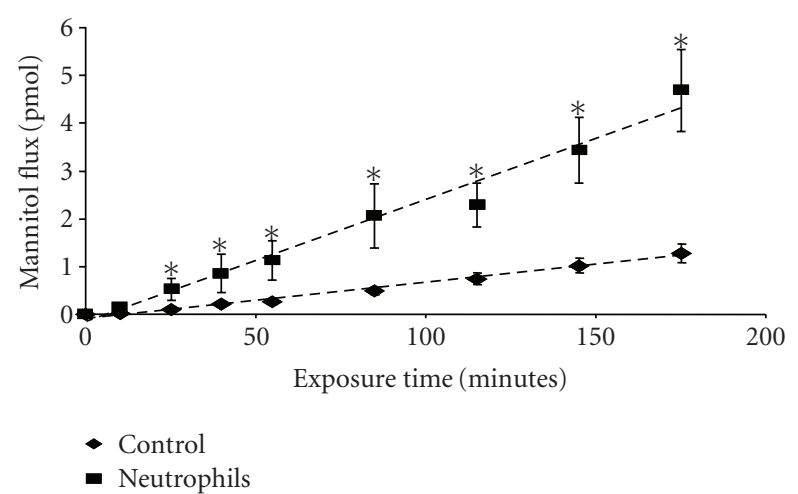

(a)

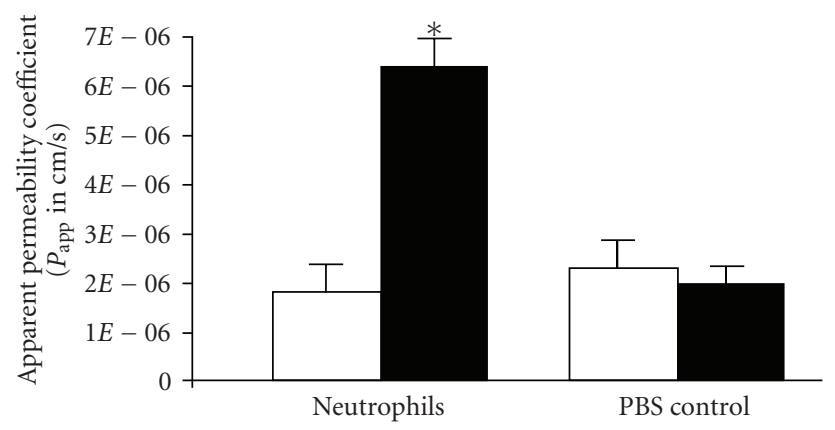

Before treatment After treatment

(b)

Figure 1: Neutrophil exposure increased explant permeability. (a) The kinetics of RPE explant permeability following neutrophil treatment. The basolateral side of the explant was incubated with neutrophils for 1 to 3 hours at $37^{\circ} \mathrm{C}$. (b) Neutrophil-induced increase of permeability of the RPE explant. The basolateral side of the explant was incubated with neutrophils for 3 hours at $37^{\circ} \mathrm{C}$. The apparent permeability was determined by $\left[{ }^{3} \mathrm{H}\right]$-mannitol flux. The increased permeability induced by neutrophils is significant compared with control. * represents $P<.05$. Addition of PBS to the explant did not change the permeability.

modified Ussing chamber method. In preliminary experiments, the dose response of neutrophils was determined and we found that the optimal dose of neutrophils for RPE barrier breakdown was $2 \times 10^{5} / \mathrm{mL}$; therefore, $2 \times 10^{5} / \mathrm{mL}$ of neutrophils were used in all subsequent experiments. The freshly prepared and washed neutrophils or vehicle alone were incubated on the basolateral side of RPE-Choroid explants. In explants receiving vehicle alone, only a low basal level increase of mannitol flux was observed over the 3 hours of observation (Figure 1(a)). By contrast, in explants exposed to neutrophils, the mannitol flux across the RPE explants was increased significantly at all time points evaluated after 20 minutes $(P<.05)$ with a maximal effect at 3 hours (Figure 1(a)). For the evaluation of apparent permeability, the explants were incubated with neutrophils for 3 hours (Figure 1(b)). A greater than 3fold increase in RPE-Choroid permeability was obtained from the explants exposed to $2 \times 10^{5} / \mathrm{mL}$ neutrophils (Figure 1(b)).

3.2. The Loss of RPE Barrier Integrity Is Associated with the Disruption of RPE Tight Junctions. To further characterize the mechanism of neutrophil-induced loss of RPE barrier integrity, the expression and localization of the tight junction-associated proteins ZO-1 (Figure 2) and occludin (Figure 3) were examined by immunofluorescent confocal microscopy. The RPE cells in the control explants showed a clear, lateral membrane staining pattern for ZO-1 and occludin and outlined the uniform polygonal shape of the RPE cells within the monolayer (Figures 2(a) and 3(a)). After 1 hour of incubation with neutrophils, the RPE explant cells showed a decrease in staining of ZO-1 and occludin (Figures 2(a) and 3(a)). Empty spaces surrounded by three or more abnormally shaped RPE cells were occasionally observed, suggesting loss of adhesion between adjacent RPE. The disruption of tight junction structure (ZO-1 and occludin) became more evident after 3 hours of neutrophil incubation (Figures 2(a) and 3(a)). Quantitative evaluation of ZO-1 immunofluorescence area demonstrated approximately $60 \%$ and $70 \%$ loss of ZO-1 staining area after 1 hour and 3 hours exposure to neutrophils, respectively (Figure 2(b), $P<.01)$. For occludin, the effect was even more striking with $75 \%$ of the immunofluorescent area of occludin staining reduced after 1 hour of neutrophil incubation, and $80 \%$ reduction after 3 hours of neutrophil incubation (Figure $3(\mathrm{~b}) ; P<.01)$. The decreased expressions of $\mathrm{ZO}-1$ and occludin induced by neutrophils also were evaluated quantitatively by western blot (Figures 2(c) and 3(c)); ZO1 protein expression decreased by $76 \%(P<.05)$ while occludin protein expression decreased by $54 \%(P<.05)$ after neutrophil exposure.

In a separate experiment, the basolateral surface of the RPE explants was exposed to neutrophil lysate, and a $75 \%$ increase in apparent permeability was found (average of 2 experiments) (Figure 4(a)), as well as a $40 \%$ and $50 \%$ decrease in ZO-1 immunoreactivity at 1 and 3 hours, respectively $(P<.05)$ (Figure $4(\mathrm{~b}))$. In each case, the effects of the neutrophil lysate were similar to but less than that found for intact neutrophils (Figure 4). These data suggest that neutrophil-induced increase in barrier permeability is associated with loss of expression of tight junction proteins occludin and ZO-1 and that at least part of this effect is mediated by a component of the neutrophil lysate.

To examine whether RPE cell death contributes to the disruption of tight junction after neutrophil treatment, the RPE cell viability was determined by PI staining. After evaluating 5000 RPE cells by FACS in each group, 382 dead cells were found in the C2-ceramide-treated group (positive control), while medium alone showed 102 dead cells and neutrophil-treated cells contained 87 dead cells (Figure 5). No difference in cell death was observed between neutrophil-treated RPE and untreated controls, suggesting 


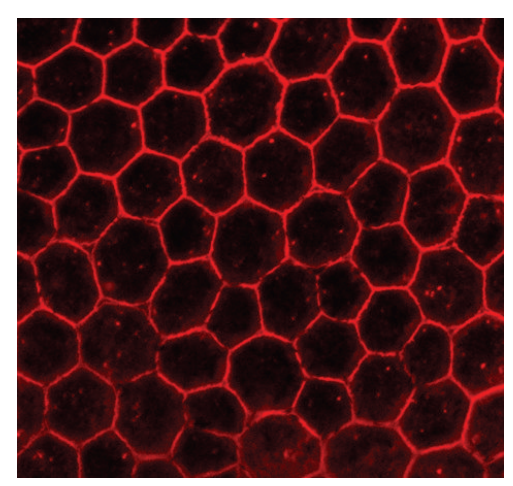

Control

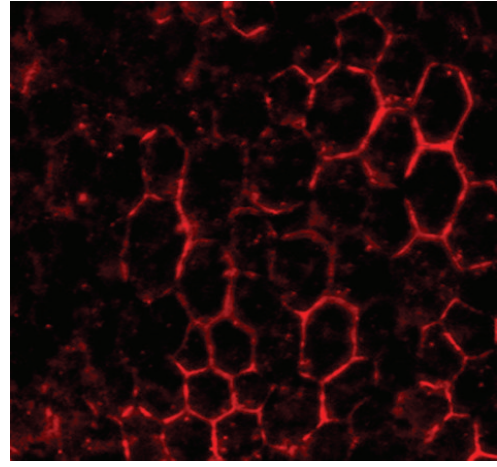

Neutrophils $1 \mathrm{hr}$

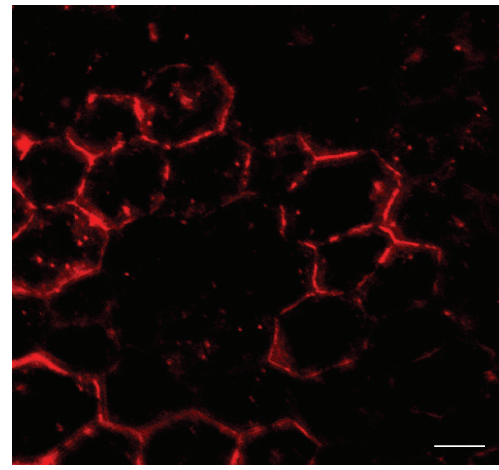

Neutrophils 3 hrs

(a)

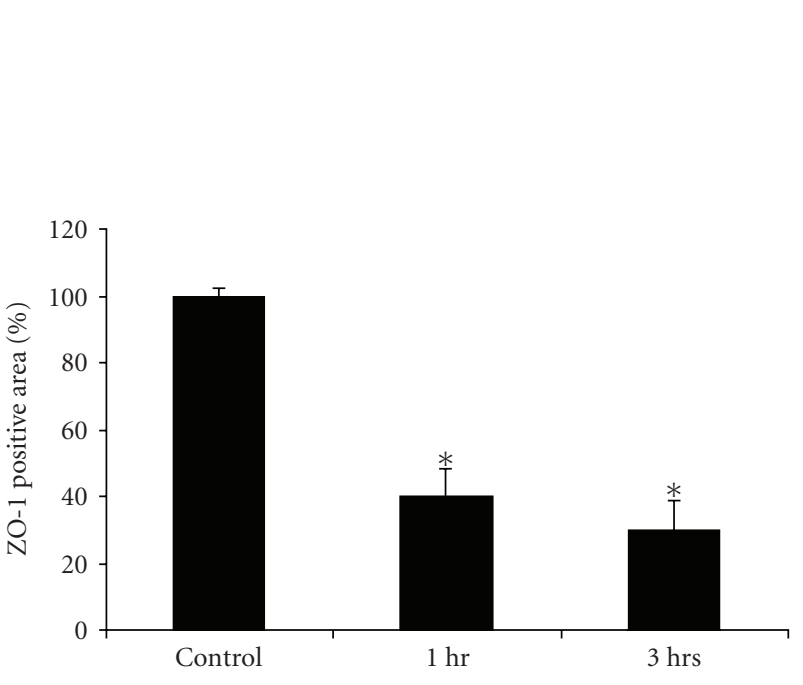

(b)

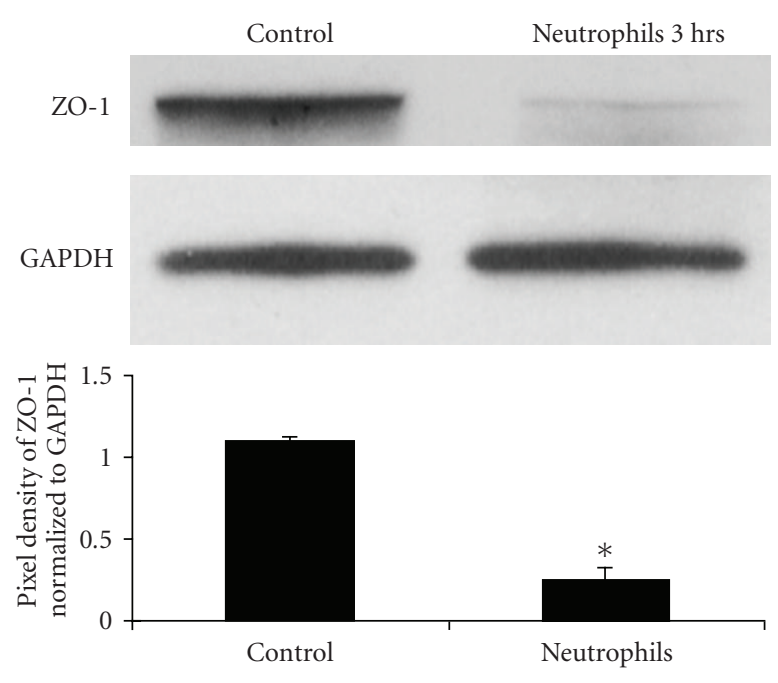

(c)

FIGURE 2: Neutrophils induced disruption of the tight junction protein ZO-1 in the RPE monolayer. (a) ZO-1 immunofluorescence staining on RPE-Choroidal explants. (b) Quantative analysis of ZO-1 immunofluorecence area on RPE-Choroidal explants. Bovine RPE-Choroidal explants were incubated with neutrophils for 1 to 3 hours at $37^{\circ} \mathrm{C}$. ZO- 1 expression was determined by confocal immunomicroscopy using anti-ZO-1 Ab. Quantitation of ZO-1 staining positive area was analyzed by using LSM 510 image software. ${ }^{*}$ represents $P<.01$. Bar $=50 \mu$ m. (c) Western blot analysis of ZO-1 expression after RPE choroidal explants exposed to neutrophils for 3 hours. In Figure 2(c), the result of densitometry from the western blot (upper panel), normalized to GAPDH, shows significant loss of ZO-1 protein, $P<.05$.

that neutrophil-induced disruption of the tight junction of the RPE monolayer was independent of significant RPE cell death. DAPI staining of the explants similarly showed that in areas of tight junction protein loss, there was no associated cell death (results not shown).

\subsection{Localization of MMP-9 in Murine Neutrophils. Previous} studies have shown that MMP-9 can cause increased permeability in retinal and lung vasculature and can alter Sertolicell tight junction dynamics [26-28]. In order to determine whether murine neutrophils, as used in this study, were a potential source of MMP-9, we evaluated MMP-9 protein expression by confocal microscopy. Approximately 30\% of the murine neutrophils showed a clear, abundant, granular cytosolic staining pattern for MMP-9 (Figure 6).
3.4. Neutrophil-Mediated Loss of RPE Barrier Integrity Is Attenuated by the Administration of Anti-MMP-9 Antibody. To determine whether MMP-9 contributes to the neutrophilinduced loss of RPE barrier integrity, RPE explant permeability was assessed in the presence of inhibitory antiMMP-9 IgG. Preliminary experiments utilizing anti-MMP IgG demonstrated prominent inhibition of MMP-9 activity by zymography when used at the concentration recommended by the manufacturer (results not shown). Goat anti-MMP-9 IgG suppressed neutrophil-induced loss of RPE permeability by approximately $65 \%(P<.05)$ (Figure 7 ). By contrast, normal goat IgG did not significantly affect neutrophil-induced increase in permeability compared to controls. These data indicate that the neutrophil-induced increase in RPE permeability was, at least partially, MMP-9 dependent. 


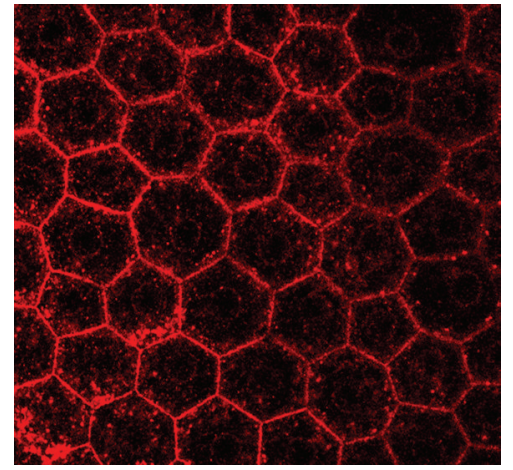

Control

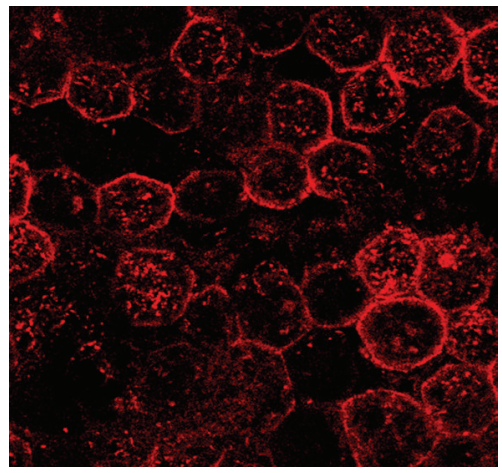

Neutrophils $1 \mathrm{hr}$

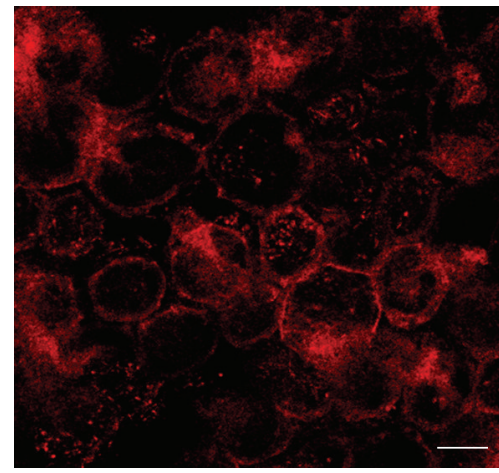

Neutrophils 3 hrs

(a)

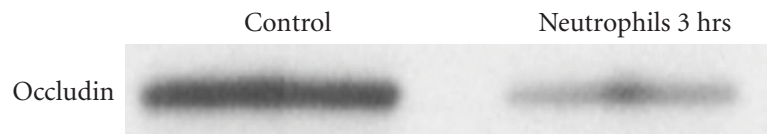

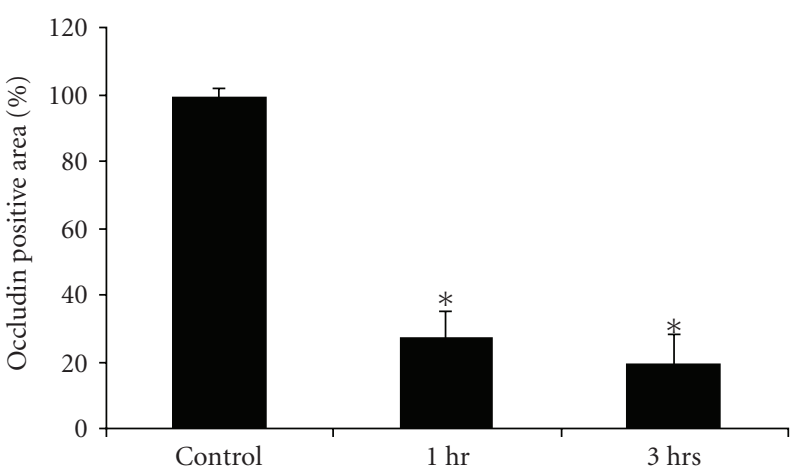

(b)
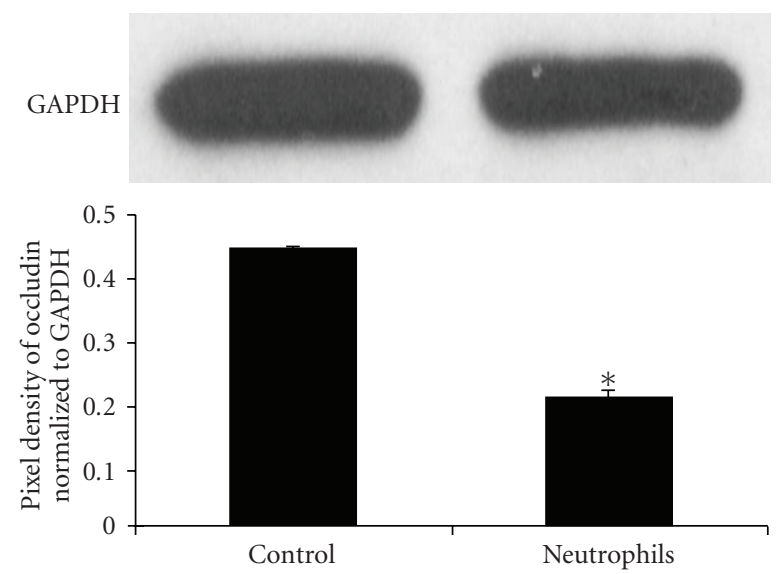

(c)

FIGURE 3: Neutrophils induced disruption of tight junction protein occludin in the RPE monolayer. (a) Occludin immunofluorescence staining on RPE-Choroidal explants. (b) Quantative analysis of occludin immunofluorecence area on RPE-Choroidal explants. Bovine RPE-Choroidal explants were incubated with neutrophils for $1-3$ hours at $37^{\circ} \mathrm{C}$. Occludin expression was determined by confocal immunomicroscopy using anti-ZO-1 Ab. Quantitation of occludin staining positive area was analyzed by using LSM 510 image software. * represents $P<.01$. Bar $=50 \mu \mathrm{m}$. (c) Western blot shows the effects of Neutrophils on occludin expression after 3-hour incubation. In (c), the result of densitometry from the western blot (upper panel), normalized to GAPDH, shows significant loss of occludin protein, $P<.05$.

\section{Discussion}

Our data show that exposure of RPE-Choroid explants to neutrophils or neutrophil lysate resulted in increased permeability across the outer BRB barrier with disruption of RPE tight junctions. Inhibition of MMP-9 inhibited the neutrophil-induced permeability indicating that this effect is mediated, in part, through MMP-9. Neutrophils are well known as executors of the acute inflammatory response where one of their major functions is to phagocytose necrotic or apoptotic cells. Evidence is emerging that neutrophils have multiple additional major functions including regulation of cellular endothelial barrier integrity $[10,12,29]$. However, the effect of neutrophils on RPE and outer BRB barrier integrity has not been directly addressed. Disruption of the outer BRB occurs in several blinding ocular diseases and in some cases is associated with infiltration of neutrophils [30]. The intriguing question is whether the neutrophil infiltration is a promoting factor of BRB disruption or only a result of a secondary bystander effect. Our study supports the notion that neutrophils contribute to the breakdown of the outer BRB barrier and suggest the possibility that inhibition of neutrophil infiltration or activity would benefit some patients with outer BRB disruption. Clinical studies have revealed that triamcinolone acetonide may be of therapeutic value in the treatment of diabetic macular edema and may provide a probarrier effect [31]. Triamcinolone acetonide acts through the NF-kB pathway to 


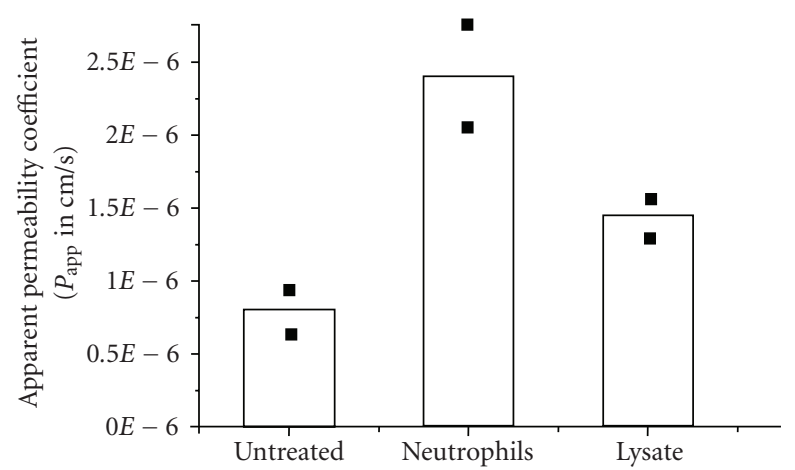

(a)

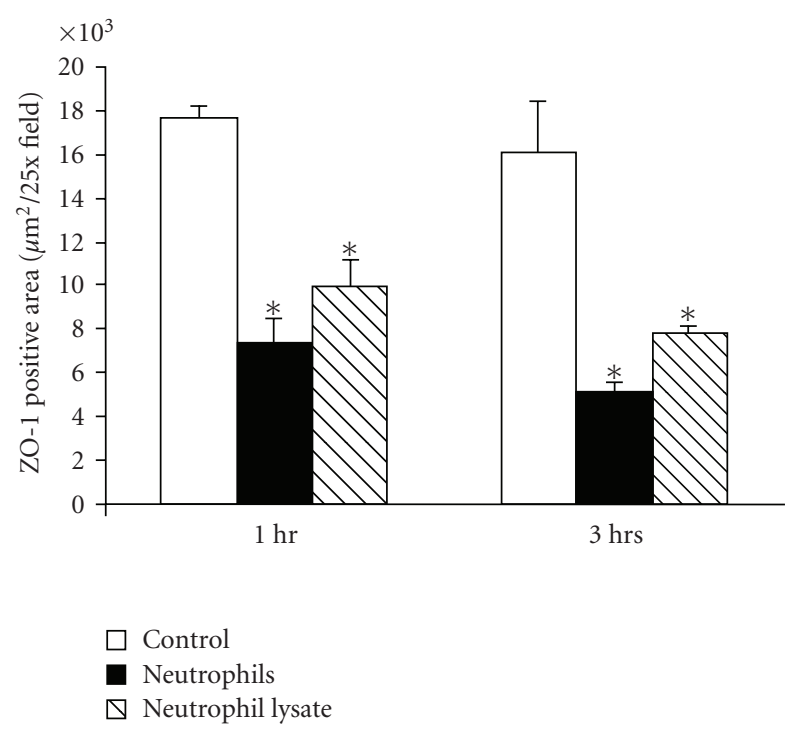

(b)

FIGURE 4: Neutrophil and neutrophil lysate exposure increased the explant permeability (a) and decreased ZO-1 (b) expression. In (a), the basolateral side of the explant was incubated with neutrophils or neutrophil lysate for 3 hours at $37^{\circ} \mathrm{C}$. The apparent permeability was determined by $\left[{ }^{3} \mathrm{H}\right]$-mannitol flux. Increased permeability induced by neutrophils or neutrophil lysate is seen compared with control (two separate experiments); the variations between experiments were less than $10 \%$. In (b), the disruption of tight junction protein ZO-1 by neutrophils or neutrophil lysate in the RPE monolayer was compared by confocal immunofluorescent staining in 3 separate experiments. Quantitation of ZO-1 staining positive area was determined by LSM 510 image software. Both neutrophil and neutrophil lysate decreased the expression of $\mathrm{ZO}-1$ expression. ${ }^{*} P<.05$

inhibit inflammatory responses, thereby leading to leukocyte apoptosis including neutrophils [32]. A recently study by Tempfer et al. shows that triamcinolone acetonide inhibits the expression of the matrix metalloproteinases MMP2, MMP8, MMP9, and MMP13 in human rotator cuff tendon cells in vitro [33]. Thus, triamcinolone acetonide could prevent loss of BRB in part by inhibiting MMPs produced by neutrophils.
One of the mediators thought to play an important role in induction of tight junction permeability is MMP-9. The role of MMP-9 in inner retinal vascular permeability has been previously evaluated; Giebel et al. demonstrated elevated MMP-9 expression in the retinas of diabetic rats, and Behzadian et al. have shown that MMP-9 plays a role in increasing retinal endothelial cell permeability induced by transforming growth factor-beta [34, 35]. While MMP-9 has been implicated in bronchial and renal epithelial permeability, the role of MMP-9 in mediating RPE permeability is relatively unexplored $[36,37]$. Our finding that MMP-9 mediates, in part, the increase in RPE permeability after neutrophil treatment provides further support for the idea that MMP-9 is an important mediator of epithelial cell permeability and disruption. Furthermore, our data indicate that neutrophils can affect RPE barrier permeability quickly with significant effects after as little as 20 minutes of incubation. While RPE themselves are a source of MMP-9 after inflammatory cytokine stimulation, the time course for this effect is much more delayed taking up to 36 hours to have a significant effect [18]. However, in the course of a prolonged acute inflammatory response in vivo, it is possible that both neutrophils and RPE could contribute to local expression of MMP-9. Previous studies have demonstrated that occludin is a proteolytic target of MMP-9 $[35,38,39]$. Interestingly, our study showed the loss of both ZO-1 and occludin; however, it is likely that $\mathrm{ZO}-1$ is not a direct target of MMP-9 but may be degraded secondary to loss of occludin.

Pretreatment with anti-MMP-9 antibody only partially blocked the increase in RPE barrier permeability caused by neutrophils. This result suggested that additional mechanisms exist by which neutrophils compromise RPE barrier integrity. Neutrophils contain an arsenal of bioactive molecules that are prestored in granules; vascular endothelial growth factor, tumor necrosis factor alpha, hepatocyte growth factor, azurocidin, and glutamate have antibarrier activities in addition to MMP-9 [12, 16, 40, 41].

In addition, we found that the effects of neutrophils on RPE permeability and the loss of tight junction protein ZO-1 were greater than those found for neutrophil lysate, which suggests that either the process of forming the cell lysate decreases activity or survival of MMP-9, or MMP-9 secreted from live cells may have greater effects on junction breakdown.

This study clearly demonstrates that neutrophils can cause disruption of outer BRB in RPE-Choroid explants. The disruption of BRB is associated with increased permeability and loss of tight junction proteins and is not a direct result of RPE cell death. The effect is mediated, at least in part by MMP-9. Together, these findings suggest that neutrophils may facilitate breakdown of the outer BRB and contribute to the pathogenesis of ocular diseases associated with increased BRB permeability. Therefore, a better understanding of the mechanisms by which neutrophils compromise RPE barrier activity will provide future therapeutic targets for blinding diseases. 


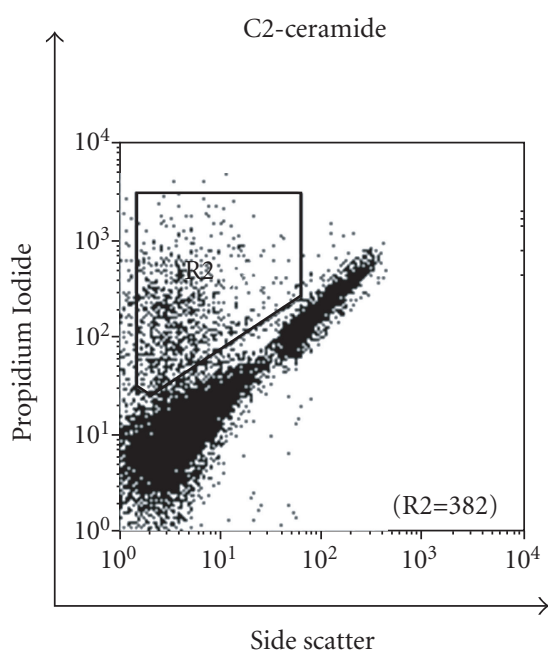

(a)

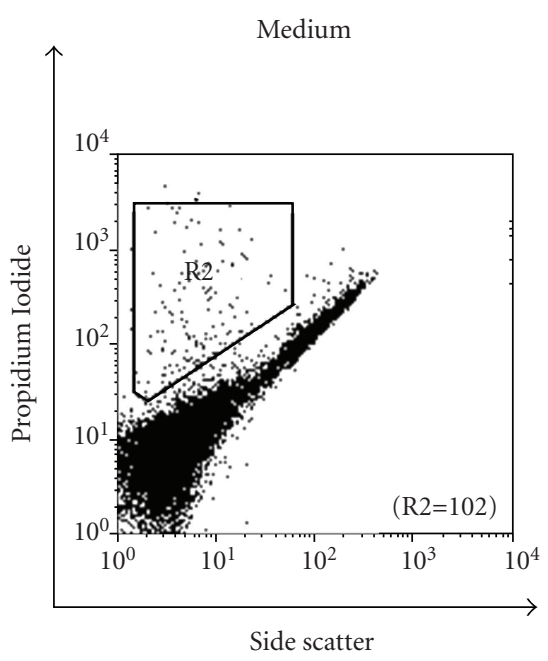

(b)

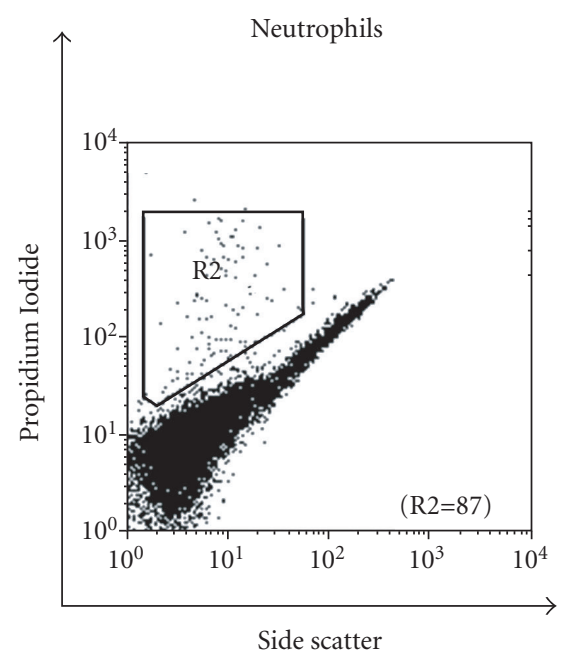

(c)

FIGURE 5: The effects of neutrophil incubation on RPEs cell death. Human RPEs were incubated with neutrophils, C2-ceramide (20 $\mu$ M; positive control), or medium (negative control) for 3 hours and then stained by Propidium iodide(PI). Cell death was evaluated by flow cytometry using 5000 cells for each experiment. The number of nonviable cells (R2) is indicated for each condition. C2-ceramide induced cell death (7.6\%). Cells incubated with neutrophils or medium alone had lower cell death, namely, $1.7 \%$ and $2.0 \%$ cell death, respectively.
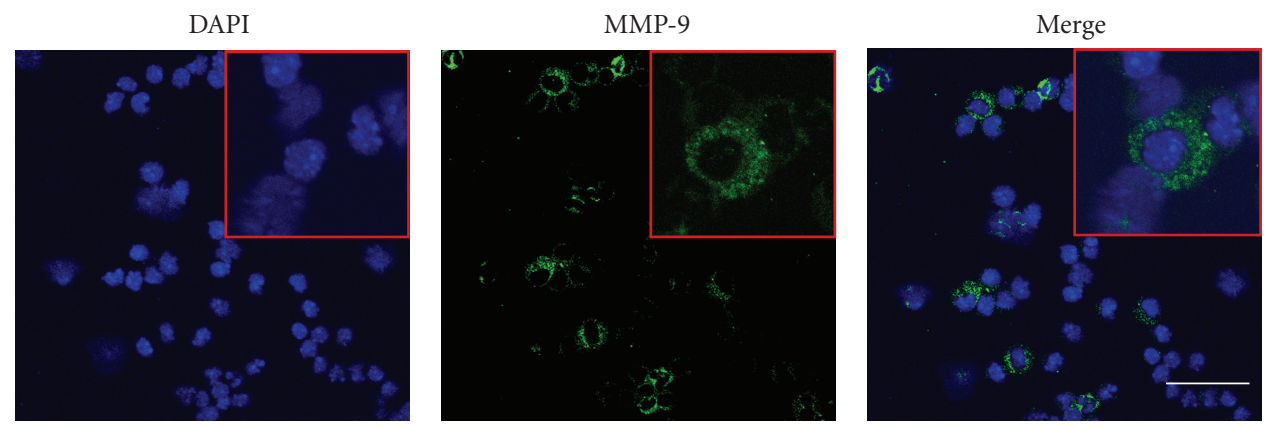

Figure 6: Localization of MMP-9 in mouse neutrophils determined by immunofluorescent confocal microscopy. DAPI, nuclear counterstained (blue); MMP-9 immunofluorescence (green). Representative neutrophils are shown at higher magnification in inset. Bar $=30 \mu \mathrm{m}$.

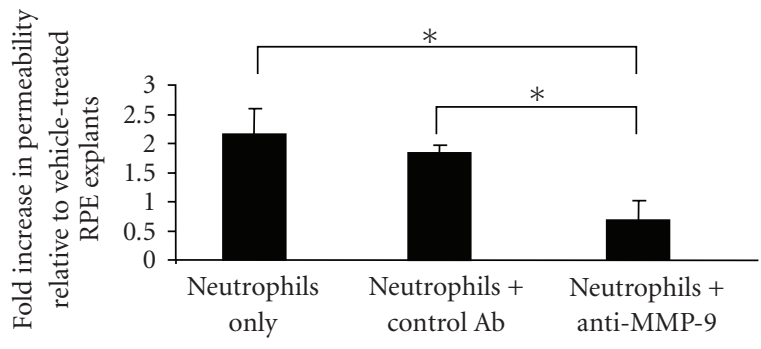

FIGURE 7: Anti-MMP-9 antibody attenuated neutrophil-mediated increase in permeability. The neutrophils were pre-treated with either anti-MMP-9 antibody or control antibody followed by incubation with explants for 3 hours at $37^{\circ} \mathrm{C}$. The apparent permeability was determined by $\left[{ }^{3} \mathrm{H}\right]$-mannitol flux and the data were presented as the fold increase in permeability relative to vehicle-treated samples. ${ }^{*}$ represents $P<.05$.

\section{Acknowledgments}

The authors thank Ernesto Barron, Xiaopeng Wang, and Anthony Rodriguez for their excellent technical assistance. This project was supported by the Arnold and Mabel Beckman Foundation and by the National Institute of Health Grants EY01545 and EY03040. Jiehao Zhou and Shikun He contributed equally to this work.

\section{References}

[1] P. A. Campochiaro, J. A. Bryan III, B. P. Conway, and E. H. Jaccoma, "Intravitreal chemotactic and mitogenic activity. Implication of blood-retinal barrier breakdown," Archives of Ophthalmology, vol. 104, no. 11, pp. 1685-1687, 1986. 
[2] E. H. Jaccoma, B. P. Conway, and P. A. Campochiaro, "Cryotherapy causes extensive breakdown of the blood-retinal barrier. A comparison with argon laser photocoagulation," Archives of Ophthalmology, vol. 103, no. 11, pp. 1728-2730, 1985.

[3] H. Canataroglu, I. Varinli, A. A. Ozcan, A. Canataroglu, F. Doran, and S. Varinli, "Interleukin (IL)-6, interleukin (IL)8 levels and cellular composition of the vitreous humor in proliferative diabetic retinopathy, proliferative vitreoretinopathy, and traumatic proliferative vitreoretinopathy," Ocular Immunology and Inflammation, vol. 13, no. 5, pp. 375-381, 2005.

[4] M. Zheng and S. S. Atherton, "Cytokine profiles and inflammatory cells during HSV-1-induced acute retinal necrosis," Investigative Ophthalmology and Visual Science, vol. 46, no. 4, pp. 1356-1363, 2005.

[5] G. Thumann, S. Hoffmann, and D. R. Hinton, "Cell biology of the retinal pigment epithelium," in Retina, S. Ryan, Ed., vol. 1, pp. 137-152, Elsevier; Mosby, London, UK, 4th edition, 2005.

[6] B. S. Fine, "Limiting membranes of the sensory retina and pigment epithelium. An electron microscopic study," Archives of Ophthalmology, vol. 66, no. 6, pp. 847-860, 1961.

[7] B. R. Stevenson, M. B. Heintzelman, J. M. Anderson, S. Citi, and M. S. Mooseker, "ZO-1 and cingulin: tight junction proteins with distsinct identities and localizations," American Journal of Physiology, vol. 257, no. 4, pp. C621-C628, 1989.

[8] B. R. Stevenson and B. H. Keon, "The tight junction: morphology to molecules," Annual Review of Cell and Developmental Biology, vol. 14, pp. 89-109, 1998.

[9] M. Jin, E. Barron, S. He, S. J. Ryan, and D. R. Hinton, "Regulation of RPE intercellular junction integrity and function by hepatocyte growth factor," Investigative Ophthalmology and Visual Science, vol. 43, no. 8, pp. 2782-2790, 2002.

[10] T. J. Williams, P. J. Jose, C. V. Wedmore, and M. J. Forrest, "Blood flow, vascular permeability and the role of neutrophils," Agents and Actions Supplements, vol. 11, pp. 39-49, 1982.

[11] C. V. Wedmore and T. J. Williams, "Control of vascular permeability by polymorphonuclear leukocytes in inflammation," Nature, vol. 289, no. 5799, pp. 646-650, 1981.

[12] V. Witko-Sarsat, P. Rieu, B. Descamps-Latscha, P. Lesavre, and L. Halbwachs-Mecarelli, "Neutrophils: molecules, functions and pathophysiological aspects," Laboratory Investigation, vol. 80, no. 5, pp. 617-654, 2000.

[13] A. M. Joussen, V. Poulaki, N. Mitsiades, et al., "Suppression of Fas-FasL-induced endothelial cell apoptosis prevents diabetic blood-retinal barrier breakdown in a model of streptozotocininduced diabetes," The FASEB Journal, vol. 17, no. 1, pp. 7678, 2003.

[14] J. Cao, D. S. McLeod, C. A. Merges, and G. A. Lutty, "Choriocapillaris degeneration and related pathologic changes in human diabetic eyes," Archives of Ophthalmology, vol. 116, no. 5, pp. 589-597, 1998.

[15] G. A. Lutty, J. Cao, and D. S. McLeod, "Relationship of polymorphonuclear leukocytes to capillary dropout in the human diabetic choroid," American Journal of Pathology, vol. 151, no. 3, pp. 707-714, 1997.

[16] J. Zhou, L. Pham, N. Zhang, et al., "Neutrophils promote experimental choroidal neovascularization," Molecular Vision, vol. 11, pp. 414-424, 2005.

[17] V. M. Elner, M. A. Burnstine, R. M. Strieter, S. L. Kunkel, and S. G. Elner, "Cell-associated human retinal pigment epithelium interleukin- 8 and monocyte chemotactic protein-1: immuno- chemical and in-situ hybridization-analyses," Experimental Eye Research, vol. 65, no. 6, pp. 781-789, 1997.

[18] S. Hoffmann, S. He, M. Ehren, S. J. Ryan, P. Wiedemann, and D. R. Hinton, "MMP-2 and MMP-9 secretion by RPE is stimulated by angiogenic molecules found in choroidal neovascular membranes," Retina, vol. 26, no. 4, pp. 454-461, 2006.

[19] S. Ikeda, H. Saito, T. Inoue, et al., "Malnutrition impairs CD11b/CD18 expression on circulating polymorphonuclear neutrophils and subsequent exudation into inflammatory sites in the early phase of glycogen-induced murine peritonitis," Journal of Parenteral and Enteral Nutrition, vol. 24, no. 5, pp. 276-279, 2000.

[20] M. S. Mulligan, A. B. Lentsch, M. Miyasaka, and P. A. Ward, "Cytokine and adhesion molecule requirements for neutrophil recruitment during glycogen-induced peritonitis," Inflammation Research, vol. 47, no. 6, pp. 251-255, 1998.

[21] N. Zhang, R. Kannan, C. T. Okamoto, S. J. Ryan, V. H. L. Lee, and D. R. Hinton, "Characterization of brimonidine transport in retinal pigment epithelium," Investigative Ophthalmology and Visual Science, vol. 47, no. 1, pp. 287-294, 2006.

[22] D. A. Frambach, J. L. Valentine, and J. J. Weiter, "Initial observations of rabbit retinal pigment epithelium-choroidsclera preparations," Investigative Ophthalmology and Visual Science, vol. 29, no. 5, pp. 814-817, 1988.

[23] H. Ueda, Y. Horibe, K.-J. Kim, and V. H. L. Lee, "Functional characterization of organic cation drug transport in the pigmented rabbit conjunctiva," Investigative Ophthalmology and Visual Science, vol. 41, no. 3, pp. 870-876, 2000.

[24] R. Kannan, M. Jin, M.-A. Gamulescu, and D. R. Hinton, "Ceramide-induced apoptosis: role of catalase and hepatocyte growth factor," Free Radical Biology and Medicine, vol. 37, no. 2, pp. 166-175, 2004.

[25] S. Sonoda, C. Spee, E. Barron, S. J. Ryan, R. Kannan, and D. R. Hinton, "A protocol for the culture and differentiation of highly polarized human retinal pigment epithelial cells," Nature protocols, vol. 4, no. 5, pp. 662-673, 2009.

[26] M. A. Behzadian, X.-L. Wang, L. J. Windsor, N. Ghaly, and R. B. Caldwell, "TGF- $\beta$ increases retinal endothelial cell permeability by increasing MMP-9: possible role of glial cells in endothelial barrier function," Investigative Ophthalmology and Visual Science, vol. 42, no. 3, pp. 853-859, 2001.

[27] M. K. Y. Siu, W. M. Lee, and C. Y. Cheng, "The interplay of collagen IV, tumor necrosis factor- $\alpha$, gelatinase B (matrix metalloprotease-9), and tissue inhibitor of metalloproteases1 in the basal lamina regulates sertoli cell-tight junction dynamics in the rat testis," Endocrinology, vol. 144, no. 1, pp. 371-387, 2003.

[28] T. Keck, J. H. Balcom IV, C. Fernández-del Castillo, B. A. Antoniu, and A. L. Warshaw, "Matrix metalloproteinase-9 promotes neutrophil migration and alveolar capillary leakage in pancreatitis-associated lung injury in the rat," Gastroenterology, vol. 122, no. 1, pp. 188-201, 2002.

[29] J. Zhou, S. A. Stohlman, D. R. Hinton, and N. W. Marten, "Neutrophils promote mononuclear cell infiltration during viral-induced encephalitis," Journal of Immunology, vol. 170, no. 6, pp. 3331-3336, 2003.

[30] I. J. Crane and J. Liversidge, "Mechanisms of leukocyte migration across the blood-retina barrier," Seminars in Immunopathology, vol. 30, no. 2, pp. 165-177, 2008.

[31] J. B. Jonas, I. Kreissig, and R. Degenring, "Intravitreal triamcinolone acetonide for treatment of intraocular proliferative, exudative, and neovascular diseases," Progress in Retinal and Eye Research, vol. 24, no. 5, pp. 587-611, 2005. 
[32] L. I. McKay and J. A. Cidlowski, "Molecular control of immune/inflammatory responses: interactions between nuclear factor- $\kappa \mathrm{B}$ and steroid receptor-signaling pathways," Endocrine Reviews, vol. 20, no. 4, pp. 435-459, 1999.

[33] H. Tempfer, R. Gehwolf, C. Lehner, et al., "Effects of crystalline glucocorticoid triamcinolone acetonide on cultered human supraspinatus tendon cells," Acta Orthopaedica, vol. 80, pp. 357-362, 2009.

[34] J. M. Gidday, Y. G. Gasche, J.-C. Copin, et al., "Leukocytederived matrix metalloproteinase- 9 mediates blood-brain barrier breakdown and is proinflammatory after transient focal cerebral ischemia," American Journal of Physiology, vol. 289, no. 2, pp. H558-H568, 2005.

[35] S. J. Giebel, G. Menicucci, P. G. McGuire, and A. Das, "Matrix metalloproteinases in early diabetic retinopathy and their role in alteration of the blood-retinal barrier," Laboratory Investigation, vol. 85, no. 5, pp. 597-607, 2005.

[36] H. Lemjabbar, P. Gosset, C. Lamblin, et al., "Contribution of $92 \mathrm{kDa}$ gelatinase/type IV collagenase in bronchial inflammation during status asthmaticus," American Journal of Respiratory and Critical Care Medicine, vol. 159, no. 4, pp. 1298-1307, 1999.

[37] A. K. Leone, J. A. Chun, C. L. Koehler, J. Caranto, and J. M. King, "Effect of proinflammatory cytokines, tumor necrosis factor- $\alpha$ and interferon- $\gamma$ on epithelial barrier function and matrix metalloproteinase- 9 in madin darby canine kidney cells," Cellular Physiology and Biochemistry, vol. 19, no. 1-4, pp. 99-112, 2007.

[38] H. Ichiyasu, J. M. McCormack, K. M. McCarthy, D. Dombkowski, F. I. Preffer, and E. E. Schneeberger, "Matrix metalloproteinase-9-deficient dendritic cells have impaired migration through tracheal epithelial tight junctions," American Journal of Respiratory Cell and Molecular Biology, vol. 30, no. 6, pp. 761-770, 2004.

[39] A. Caron, R. R. Desrosiers, and R. Béliveau, "Ischemia injury alters endothelial cell properties of kidney cortex: stimulation of MMP-9," Experimental Cell Research, vol. 310, no. 1, pp. 105-116, 2005.

[40] N. Gautam, A. M. Olofsson, H. Herwald, et al., "Heparinbinding protein (HBP/CAP37): a missing link in neutrophilevoked alteration of vascular permeability," Nature Medicine, vol. 7, no. 10, pp. 1123-1127, 2001.

[41] C. D. Collard, K. A. Park, M. C. Montalto, et al., "Neutrophilderived glutamate regulates vascular endothelial barrier function," Journal of Biological Chemistry, vol. 277, no. 17, pp. 14801-14811, 2002. 

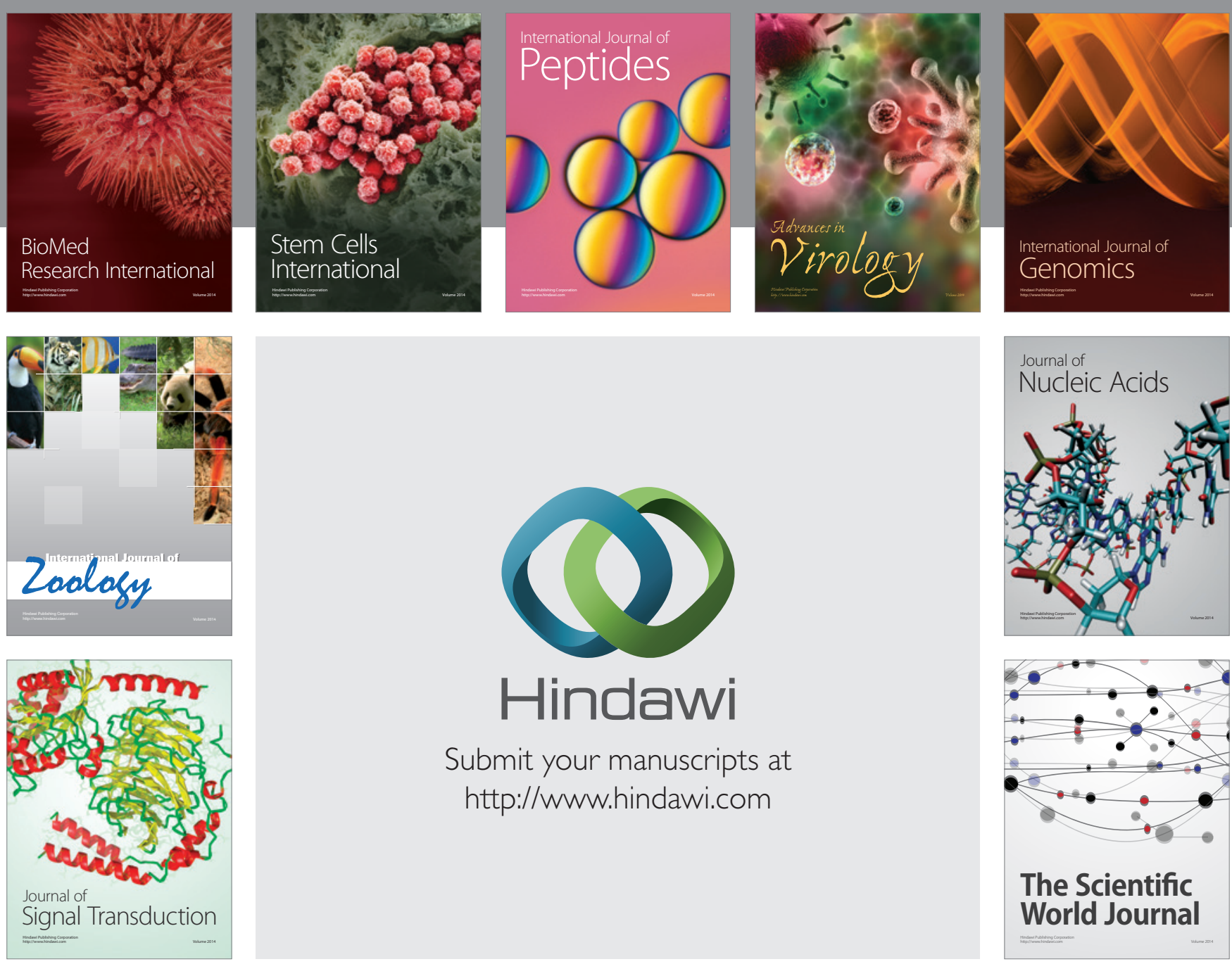

Submit your manuscripts at

http://www.hindawi.com
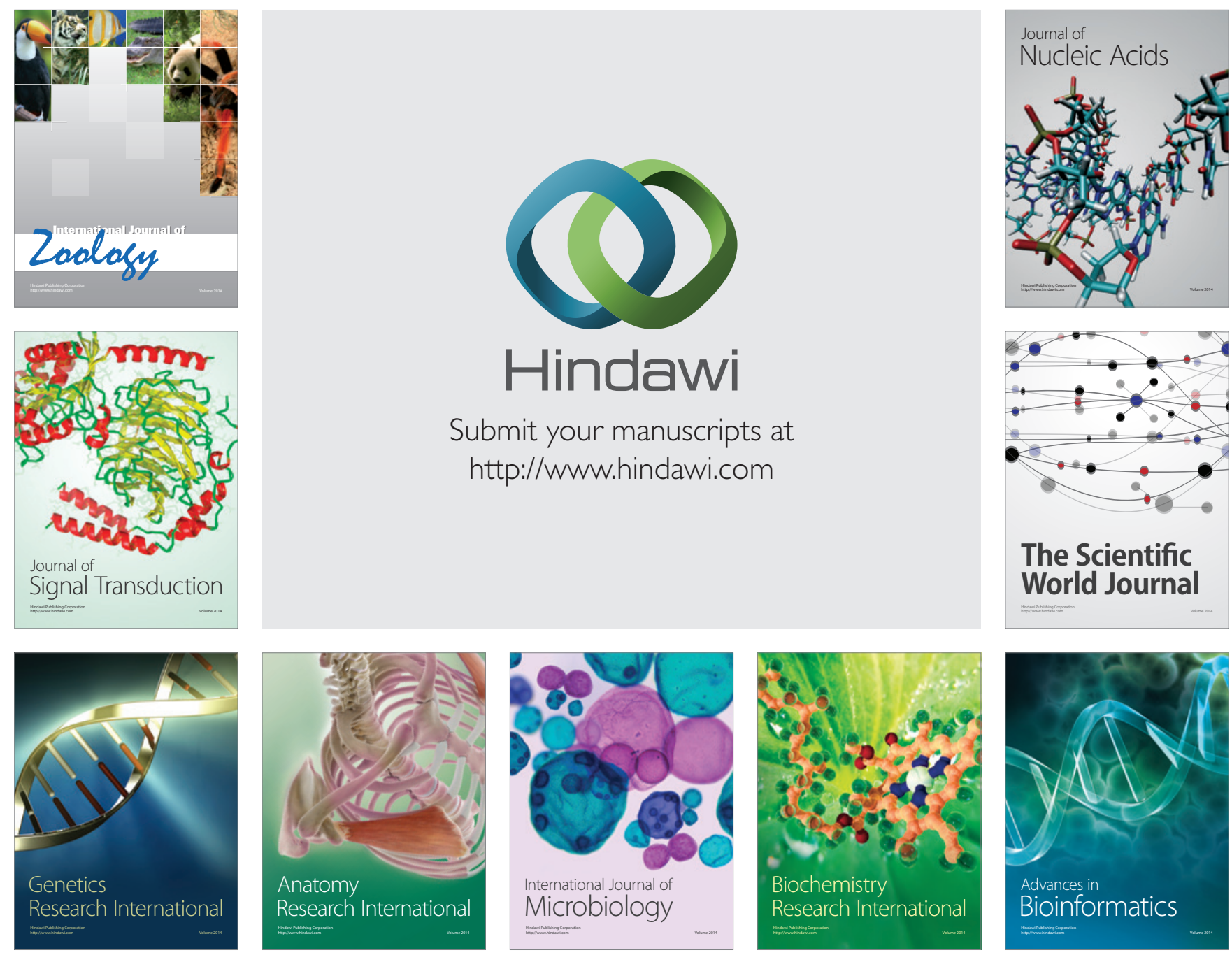

The Scientific World Journal
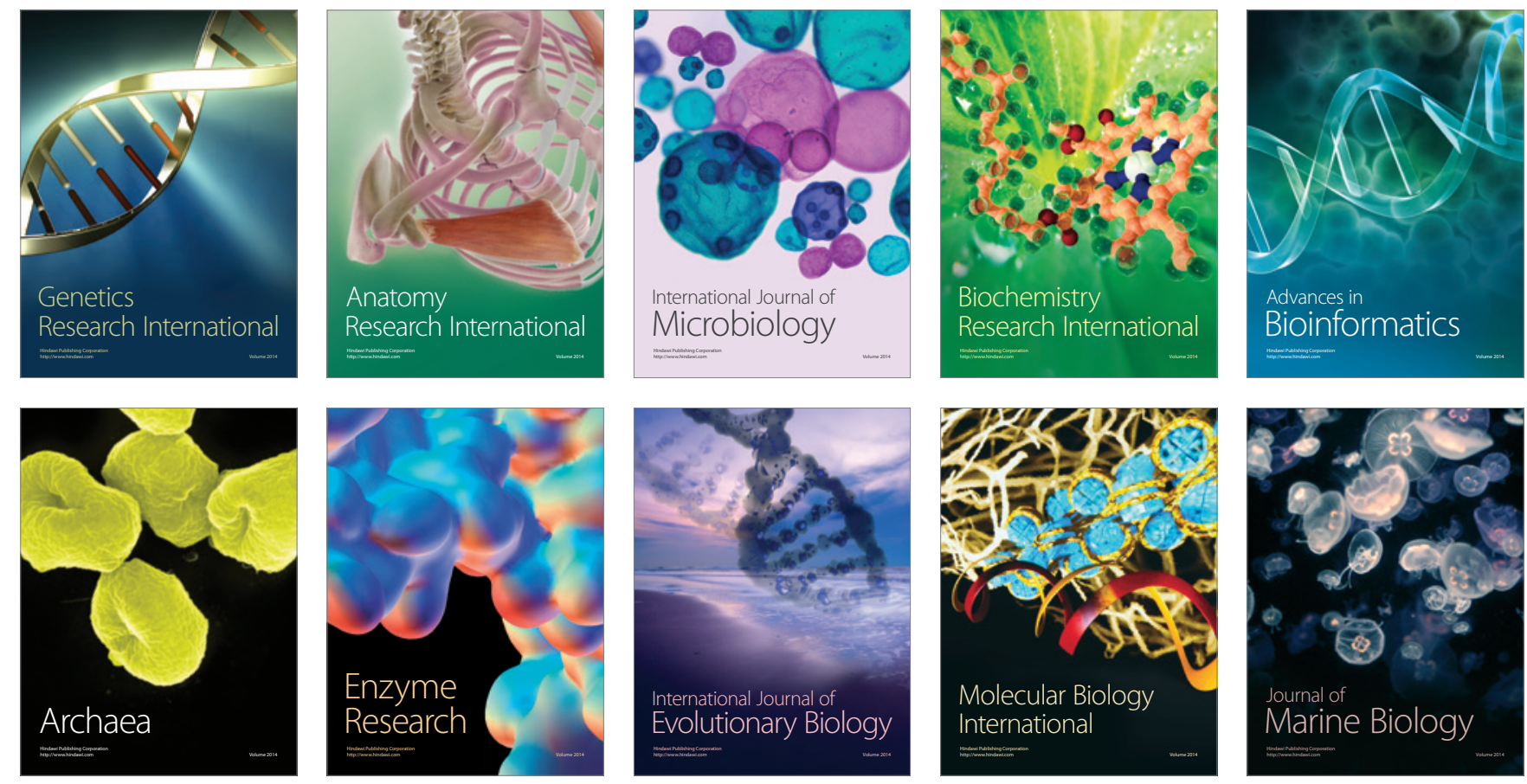